\title{
Mixed methods for a stream-function - vorticity formulation of the axisymmetric Brinkman equations
}

\author{
Verónica Anaya • David Mora • Carlos Reales · \\ Ricardo Ruiz-Baier
}

Received: date / Accepted: date

\begin{abstract}
This paper is devoted to the numerical analysis of a family of finite element approximations for the written in terms of the stream-function and vorticity. A mixed formulation is introduced involving appropriate weighted Sobolev spaces, numerical examples are presented to illustrate the convergence and performance of the proposed schemes.
\end{abstract}

Keywords Brinkman equations - Stream-function and vorticity formulation · Axisymmetric domains · Finite element method · Stability analysis · Error estimates.

Mathematics Subject Classification (2000) 65N30 65 N12 $\cdot 76$ D07 65 N15 65 J20

\section{Introduction}

. In addition, a manipulation of the equations permits to eliminate the pressure from the formulation. However, if pressure profiles are required, they can be recovered via a generalized Poisson problem with a datum coming from the stream-function solution (in a similar spirit to the decoupled methods recently proposed in [22,7] for Brinkman equations in Cartesian coordinates).

studies involving different numerical methods for axisymmetric (viscous or non-viscous) flows (see e.g. [3, 5, 7, 8, 10, 14, 16, 20, 23, 33. and the references therein). More precisely, in the recent contribution 4 the authors propose a spectral method for a stream-function vorticity formulation of the Stokes equations, where the cylindrical symmetry reduces a three-dimensional problem to a bidimensional one. the analysis of existence and uniqueness of continuous and discrete solutions is established using standard arguments for saddle-point problems (see [21]), and we propose a finite element discretization based on piecewise polynomials of order $k \geq 1$ for all scalar fields, defined on triangular meshes. This method represents only six degrees of freedom per element, decoupled from a pressure

V. Anaya

GIMNAP, Departamento de Matemática, Universidad del Bío-Bío, Concepción, Chile. E-mail: vanaya@ubiobio.cl

D. Mora

GIMNAP, Departamento de Matemática, Universidad del Bío-Bío, Concepción, Chile; and Centro de Investigación en Ingeniería Matemática ( $\left.\mathrm{CI}^{2} \mathrm{MA}\right)$, Universidad de Concepción, Concepción, Chile. E-mail: dmora@ubiobio.cl

C. Reales $(\bowtie)$

Departamento de Matemáticas y Estadística, Universidad de Córdoba, Montería, Colombia. E-mail: creales@correo.unicordoba.edu.co

R. Ruiz-Baier

Mathematical Institute, Oxford University, A. Wiles Building, Radcliffe Observatory Quarter, Woodstock Road, OX2 6GG Oxford, UK. E-mail: ruizbaier@maths.ox.ac.uk 
solve (approximated in axisymmetric $H^{1}$-conforming spaces and having three degrees of freedom per element), thus being a very competitive scheme, for instance, less expensive than the mixed method recently introduced in [6]. Our optimal order error estimates are derived from the continuous dependence on the data and an appropriate Céa estimate, Moreover, a duality argument allows us to improve the order of convergence of the vorticity and the stream-function approximations in $L^{2}$-norm.

The remainder of this paper is structured as follows. Section 2 collects the relevant formulations of the Brinkman problem, for velocity and pressure in Cartesian coordinates, its reduction to the axisymmetric case, and a stream-function-vorticity form. The weak formulation, along with some preliminary results are also presented. In Section 3, we prove the unique solvability and stability properties of the proposed formulation. In Section 4, we introduce the finite element discretization of our variational formulation, for which we prove a discrete inf-sup condition uniformly with respect to the fluid viscosity $\nu$ and the mesh parameter $h$; moreover, we establish optimal error estimates. Some illustrative numerical tests are postponed to Section 5. We close with a few remarks and perspectives in Section 6 .

\section{Formulations of the linear Brinkman equations in different coordinates}

\subsection{Cartesian coordinates}

The linear Brinkman equations govern the motion of an incompressible viscous fluid within a porous medium. The system is

$$
\begin{aligned}
\breve{\mathbf{K}}^{-1} \breve{\boldsymbol{u}}-\nu \Delta \breve{\boldsymbol{u}}+\nabla \breve{p}=\breve{\boldsymbol{f}} & \text { in } \breve{\Omega}, \\
\operatorname{div} \breve{\boldsymbol{u}}=0 & \text { in } \breve{\Omega}, \\
\breve{\boldsymbol{u}} \cdot \breve{\boldsymbol{n}}=0 & \text { on } \partial \breve{\Omega}, \\
\operatorname{curl} \breve{\boldsymbol{u}} \times \breve{\boldsymbol{n}}=0 & \text { on } \partial \breve{\Omega}
\end{aligned}
$$

where $\breve{\Omega} \subset \mathbb{R}^{3}$ is a given spatial domain. The sought quantities are the local volume-average velocity $\breve{\boldsymbol{u}}$ and the pressure field $\breve{p}$. The permeability $\breve{\mathbf{K}}$ is a symmetric and positive definite tensor, and without loss of generality we can restrict ourselves to the isotropic case where the inverse permeability distribution can be represented by a scalar function, i.e. $\breve{\mathbf{K}}^{-1}=\breve{\sigma} \mathbf{I}$. The inverse permeability has $\mathrm{L}^{\infty}(\breve{\Omega})$ regularity, with $\breve{\sigma}_{\min } \leq \breve{\sigma}(x, y, z) \leq \breve{\sigma}_{\max }$ a.e. in $\breve{\Omega}$. For simplicity, we assume a positive fluid viscosity $0<\nu \leq \nu_{\max }$.

\subsection{Axisymmetric case}

Under axial symmetry of the domain, the forcing term, and the inverse permeability, we can replace them by $\Omega, \boldsymbol{f}$, and $\sigma$, respectively, with $0<\sigma_{\min } \leq \sigma(r, z) \leq \sigma_{\max }$ a.e. in $\Omega$, and system $(2.1 \mathrm{a})-(2.1 \mathrm{~d})$ can be recast as a problem

Moreover, if we introduce a vorticity field, scaled with respect to viscosity, $\omega=\sqrt{\nu} \operatorname{rot} \boldsymbol{u}$, we arrive at the following problem

$$
\begin{aligned}
& \sigma \boldsymbol{u}+\sqrt{\nu} \operatorname{curl}_{\mathbf{a}} \omega+\nabla p=\boldsymbol{f} \quad \text { in } \Omega, \\
& \omega-\sqrt{\nu} \operatorname{rot} \boldsymbol{u}=0 \quad \text { in } \Omega \text {, } \\
& \operatorname{div}_{\mathrm{a}} \boldsymbol{u}=0 \quad \text { in } \Omega, \\
& \boldsymbol{u} \cdot \boldsymbol{n}=0 \quad \text { on } \Gamma, \\
& \omega=0 \quad \text { on } \Gamma \text {, }
\end{aligned}
$$


where axisymmetric counterparts of the usual differential operators acting on vectors and scalars employed herein read

$$
\begin{gathered}
\operatorname{div}_{\mathrm{a}} \boldsymbol{v}:=\partial_{r} v_{r}+r^{-1} v_{r}+\partial_{z} v_{z}, \quad \operatorname{rot} \boldsymbol{v}:=\partial_{r} v_{z}-\partial_{z} v_{r} \\
\operatorname{curl}_{\mathbf{a}} \varphi:=\left(\partial_{z} \varphi,-\partial_{r} \varphi-\frac{1}{r} \varphi\right)^{\mathrm{t}}
\end{gathered}
$$

2.3 Axisymmetric stream-function-vorticity formulation

Next, we realize that the incompressibility condition $(2.2 \mathrm{c}$ ) is equivalent to the existence of a scalar stream-function $\psi$ satisfying $\boldsymbol{u}=\operatorname{curl}_{\mathbf{a}} \psi$, with $\psi=0$ on $\Gamma$ (cf. Lemma1] and [4]24]).

\subsection{Recurrent notation and auxiliary results}

Before stating a weak form to (2.3), we recall some standard definitions of weighted Sobolev spaces and involved norms (see further details in e.g. [26]). Let $\mathrm{L}_{\alpha}^{p}(\Omega)$ denote the weighted Lebesgue space of all measurable functions $\varphi$ defined in $\Omega$ for which

$$
\|\varphi\|_{\mathrm{L}_{\alpha}^{p}(\Omega)}^{p}:=\int_{\Omega}|\varphi|^{p} r^{\alpha} \mathrm{d} r \mathrm{~d} z<\infty .
$$

The subspace $\mathrm{L}_{1,0}^{2}(\Omega)$ of $\mathrm{L}_{1}^{2}(\Omega)$ contains functions $q$ with zero weighted integral $(q, 1)_{r, \Omega}=0$, where

$$
(s, t)_{r, \Omega}:=\int_{\Omega} \operatorname{str} \mathrm{d} r \mathrm{~d} z,
$$

for all sufficiently regular functions $s, t$. The weighted Sobolev space $\mathrm{H}_{1}^{k}(\Omega)$ consists of all functions in $\mathrm{L}_{1}^{2}(\Omega)$ whose derivatives up to order $k$ are also in $\mathrm{L}_{1}^{2}(\Omega)$. This space is provided with semi-norms and norms defined in the standard way; in particular,

$$
|\varphi|_{\mathrm{H}_{1}^{1}(\Omega)}^{2}:=\int_{\Omega}\left(\left|\partial_{r} \varphi\right|^{2}+\left|\partial_{z} \varphi\right|^{2}\right) r \mathrm{~d} r \mathrm{~d} z
$$

is a norm onto the Hilbert space $\mathrm{H}_{1}^{1}(\Omega) \cap \mathrm{L}_{1,0}^{2}(\Omega)$. Furthermore, the space $\widetilde{\mathrm{H}}_{1}^{1}(\Omega):=\mathrm{H}_{1}^{1}(\Omega) \cap \mathrm{L}_{-1}^{2}(\Omega)$ is endowed with the following norm and semi-norm, respectively (the former being $\nu$-dependent):

$$
\begin{gathered}
\|\varphi\|_{\widetilde{\mathrm{H}}_{1}^{1}(\Omega)}:=\left(\|\varphi\|_{\mathrm{L}_{1}^{2}(\Omega)}^{2}+\nu|\varphi|_{\mathrm{H}_{1}^{1}(\Omega)}^{2}+\nu\|\varphi\|_{\mathrm{L}_{-1}^{2}(\Omega)}^{2}\right)^{1 / 2}, \\
\|\varphi\|_{\widetilde{\mathrm{H}}_{1}^{1}(\Omega)}:=\left(|\varphi|_{\mathrm{H}_{1}^{1}(\Omega)}^{2}+\|\varphi\|_{\mathrm{L}_{-1}^{2}(\Omega)}^{2}\right)^{1 / 2} .
\end{gathered}
$$

We will also require the following weighted scalar and vectorial functional spaces:

$$
\begin{aligned}
\mathrm{H}_{1, \diamond}^{1}(\Omega) & :=\left\{\varphi \in \mathrm{H}_{1}^{1}(\Omega) ; \varphi=0 \text { on } \Gamma\right\}, \\
\widetilde{\mathrm{H}}_{1, \diamond}^{1}(\Omega) & :=\left\{\varphi \in \widetilde{\mathrm{H}}_{1}^{1}(\Omega) ; \varphi=0 \text { on } \Gamma\right\}, \\
\mathbf{H}\left(\operatorname{div}_{\mathrm{a}}, \Omega\right) & :=\left\{\boldsymbol{v} \in \mathrm{L}_{1}^{2}(\Omega)^{2} ; \operatorname{div}_{\mathrm{a}} \boldsymbol{v} \in \mathrm{L}_{1}^{2}(\Omega)\right\}, \\
\mathrm{H}\left(\operatorname{curl}_{\mathbf{a}}, \Omega\right) & :=\left\{\varphi \in \mathrm{L}_{1}^{2}(\Omega) ; \operatorname{curl}_{\mathbf{a}} \varphi \in \mathrm{L}_{1}^{2}(\Omega)^{2}\right\}, \\
\mathbf{H}(\operatorname{rot}, \Omega) & :=\left\{\boldsymbol{v} \in \mathrm{L}_{1}^{2}(\Omega)^{2} ; \operatorname{rot} \boldsymbol{v} \in \mathrm{L}_{1}^{2}(\Omega)\right\} .
\end{aligned}
$$


We observe that as a consequence of [25, Proposition 2.1], the entity in 2.4 is a norm in $\widetilde{\mathrm{H}}_{1, \diamond}^{1}(\Omega)$. In addition, the spaces $\mathbf{H}\left(\operatorname{div}_{\mathrm{a}}, \Omega\right)$ and $\mathrm{H}\left(\mathbf{c u r l}_{\mathbf{a}}, \Omega\right)$ are endowed respectively by the norms:

$$
\begin{aligned}
\|\boldsymbol{v}\|_{\mathbf{H}\left(\operatorname{div}_{\mathbf{a}}, \Omega\right)} & :=\left(\|\boldsymbol{v}\|_{\mathrm{L}_{1}^{2}(\Omega)^{2}}^{2}+\left\|\operatorname{div}_{\mathbf{a}} \boldsymbol{v}\right\|_{\mathrm{L}_{1}^{2}(\Omega)}^{2}\right)^{1 / 2}, \\
\|\varphi\|_{\mathrm{H}\left(\operatorname{curl}_{\mathbf{a}}, \Omega\right)}: & :=\left(\|\varphi\|_{\mathrm{L}_{1}^{2}(\Omega)}^{2}+\nu\left\|\operatorname{curl}_{\mathbf{a}} \varphi\right\|_{\mathrm{L}_{1}^{2}(\Omega)^{2}}^{2}\right)^{1 / 2}, \\
\|\varphi\|_{\mathrm{H}\left(\operatorname{curl}_{\mathbf{a}}, \Omega\right)} & :=\left\|\operatorname{curl}_{\mathbf{a}} \varphi\right\|_{\mathrm{L}_{1}^{2}(\Omega)^{2}} .
\end{aligned}
$$

Moreover, it holds that

$$
\begin{gathered}
\|\varphi\|_{\widetilde{\mathrm{H}}_{1}^{1}(\Omega)} \leq\|\varphi\|_{\mathrm{H}\left(\operatorname{curl}_{\mathbf{a}}, \Omega\right)} \leq \sqrt{2}\|\varphi\|_{\widetilde{\mathrm{H}}_{1}^{1}(\Omega)} \quad \forall \varphi \in \widetilde{\mathrm{H}}_{1}^{1}(\Omega), \\
\|\varphi\|_{\widetilde{\mathrm{H}}_{1}^{1}(\Omega)} \leq\|\varphi\|_{\mathrm{H}\left(\operatorname{curl}_{\mathrm{a}}, \Omega\right)} \leq \sqrt{2}\|\varphi\|_{\widetilde{\mathrm{H}}_{1}^{1}(\Omega)} \quad \forall \varphi \in \widetilde{\mathrm{H}}_{1, \diamond}^{1}(\Omega) .
\end{gathered}
$$

The following result .

Lemma 1 Let $\Omega$ be simply connected. For any $s>1$, if $\boldsymbol{v} \in\left[\widetilde{\mathrm{H}}_{1, \diamond}^{1}(\Omega) \cap \mathrm{H}_{1}^{s}(\Omega)\right]^{2}$ satisfies $\operatorname{div}_{\mathrm{a}} \boldsymbol{v}=0$, and $\boldsymbol{v} \cdot \boldsymbol{n}=0$ on $\Gamma$, then there exists a unique potential $\varphi \in \mathrm{H}_{1}^{s+1}(\Omega)$ such that $\boldsymbol{v}=\operatorname{curl}_{\mathbf{a}} \varphi$, and $\varphi=0$ on $\Gamma$.

On the other hand, let $H_{1}^{1 / 2}(\Gamma)$ be the trace space associated to $\mathrm{H}_{1}^{1}(\Omega)$, and notice that the normal trace operator on $\Gamma$ is defined by $\left.\boldsymbol{v} \mapsto \boldsymbol{v} \cdot \boldsymbol{n}\right|_{\Gamma}$, and it is continuous from $\mathbf{H}\left(\operatorname{div}_{\mathrm{a}}, \Omega\right)$ into the dual space of $H_{1}^{1 / 2}(\Gamma)$. We next recall the following Green identities ().

Lemma 2 For any $\boldsymbol{v} \in \mathbf{H}\left(\operatorname{div}_{\mathrm{a}}, \Omega\right)$ and $q \in \mathrm{H}_{1}^{1}(\Omega)$, the following Green formula holds

$$
\left(\operatorname{div}_{\mathrm{a}} \boldsymbol{v}, q\right)_{r, \Omega}+(\boldsymbol{v}, \nabla q)_{r, \Omega}=\langle\boldsymbol{v} \cdot \boldsymbol{n}, q\rangle_{r, \Gamma} .
$$

Lemma 3 For any $\boldsymbol{v} \in \mathbf{H}(\operatorname{rot}, \Omega)$ and $\varphi \in \widetilde{\mathrm{H}}_{1}^{1}(\Omega)$, we have the following Green formula

$$
\left(\boldsymbol{v}, \operatorname{curl}_{\mathbf{a}} \varphi\right)_{r, \Omega}-(\varphi, \operatorname{rot} \boldsymbol{v})_{r, \Omega}=\langle\boldsymbol{v} \cdot \boldsymbol{t}, \varphi\rangle_{r, \Gamma} .
$$

\subsection{The variational formulation}

Then, combining Lemmas 2 and 3 with a direct application of the boundary conditions, yields the following variational problem: Find $(\psi, \omega) \in \widetilde{\mathrm{H}}_{1, \diamond}^{1}(\Omega) \times \widetilde{\mathrm{H}}_{1, \diamond}^{1}(\Omega)$ such that

$$
\begin{array}{ll}
a(\psi, \varphi)+b(\varphi, \omega)=F(\varphi) & \forall \varphi \in \widetilde{\mathrm{H}}_{1, \diamond}^{1}(\Omega), \\
b(\psi, \theta)-d(\omega, \theta)=0 & \forall \theta \in \widetilde{\mathrm{H}}_{1, \diamond}^{1}(\Omega),
\end{array}
$$

where the involved bilinear forms and linear functional are

$$
\begin{aligned}
a(\psi, \varphi) & :=\left(\sigma \operatorname{curl}_{\mathbf{a}} \psi, \operatorname{curl}_{\mathbf{a}} \varphi\right)_{r, \Omega}, \quad b(\varphi, \omega):=\left(\sqrt{\nu} \operatorname{curl}_{\mathbf{a}} \omega, \operatorname{curl}_{\mathbf{a}} \varphi\right)_{r, \Omega}, \\
d(\omega, \theta) & :=(\omega, \theta)_{r, \Omega}, \quad F(\varphi):=\left(\boldsymbol{f}, \operatorname{curl}_{\mathbf{a}} \varphi\right)_{r, \Omega} .
\end{aligned}
$$

Remark 1 The discussion about possible shortcomings of the boundary treatment (??), (??) and the associated issues in representing no-slip velocity conditions or other wall laws is not part of the goals of this paper. We refer the interested reader to [11,13,34,7]. However, we do stress that imposition of tangential velocities poses no difficulty in our framework. For instance, if we want to set $\boldsymbol{u} \cdot \boldsymbol{t}=u^{t}$ with a known $u^{t}$ on $\Gamma_{t} \subset \Gamma$, then Lemma 3 suggests that the adequate test space for the vorticity field would be

Also from Lemma 3 it follows that a non-homogeneous term

$$
H(\theta):=\left\langle\sqrt{\nu} u^{t}, \theta\right\rangle_{r, \Gamma_{t}} \quad \forall \theta \in \tilde{\mathrm{H}}_{1, t}^{1}(\Omega),
$$

should appear in the second equation of 2.7 . 


\section{Well-posedness of the continuous problem}

In this section, we prove that the continuous variational formulation (2.7) is uniquely solvable. With this aim, we recall the following abstract result (see e.g. [21, Theorem 1.3]):

Theorem 1 Let $\left(\mathcal{X},\langle\cdot, \cdot\rangle_{\mathcal{X}}\right)$ be a Hilbert space. Let $\mathcal{A}: \mathcal{X} \times \mathcal{X} \rightarrow \mathbb{R}$ be a bounded symmetric bilinear form, and let $\mathcal{G}: \mathcal{X} \rightarrow \mathbb{R}$ be a bounded functional. Assume that there exists $\bar{\beta}>0$ such that

$$
\sup _{\substack{y \in \mathcal{X} \\ y \neq 0}} \frac{\mathcal{A}(x, y)}{\|y\|_{\mathcal{X}}} \geq \bar{\beta}\|x\|_{\mathcal{X}} \quad \forall x \in \mathcal{X} .
$$

Then, there exists a unique $x \in \mathcal{X}$, such that

$$
\mathcal{A}(x, y)=\mathcal{G}(y) \quad \forall y \in \mathcal{X} .
$$

Moreover, there exists $C>0$, independent of the solution, such that

$$
\|x\|_{\mathcal{X}} \leq C\|\mathcal{G}\|_{\mathcal{X}^{\prime}}
$$

Theorem 2 The variational problem (2.7) admits a unique solution $(\psi, \omega) \in \widetilde{\mathrm{H}}_{1, \diamond}^{1}(\Omega) \times \widetilde{\mathrm{H}}_{1, \diamond}^{1}(\Omega)$. Moreover, there exists $C>0$ independent of $\nu$ such that

$$
\|\psi\|_{\widetilde{\mathrm{H}}_{1}^{1}(\Omega)}+\|\omega\|_{\widetilde{\mathrm{H}}_{1}^{1}(\Omega)} \leq C\|\boldsymbol{f}\|_{\mathrm{L}_{1}^{2}(\Omega)^{2}} .
$$

Proof First, we define $\mathcal{X}:=\widetilde{\mathrm{H}}_{1, \diamond}^{1}(\Omega) \times \widetilde{\mathrm{H}}_{1, \diamond}^{1}(\Omega)$ (endowed with the corresponding product norm: $\|\cdot\|_{\widetilde{\mathrm{H}}_{1}^{1}(\Omega)}$ and $\|\cdot\|_{\widetilde{\mathrm{H}}_{1}^{1}(\Omega)}$, respectively) and the following bilinear form and linear functional:

$$
\mathcal{A}((\psi, \omega),(\varphi, \theta)):=a(\psi, \varphi)+b(\varphi, \omega)+b(\psi, \theta)-d(\omega, \theta), \quad \mathcal{G}((\varphi, \theta)):=F(\varphi) .
$$

To continue, it suffices to verify the hypotheses of Theorem 1 . First, we note that the linear functional $\mathcal{G}(\cdot)$ is bounded and as a consequence of the boundedness of $a(\cdot, \cdot) b(\cdot, \cdot)$, and $d(\cdot, \cdot)$, one has that the bilinear form $\mathcal{A}(\cdot, \cdot)$ is also bounded with constants independent of $\nu$.

The next step consists in proving that the bilinear form $\mathcal{A}(\cdot, \cdot)$ satisfies the inf-sup condition 3.1. With this aim, we have that for any $(\psi, \omega) \in \mathcal{X}$, we define

$$
\tilde{\varphi}:=(\psi+\hat{c} \sqrt{\nu} \omega) \in \widetilde{\mathrm{H}}_{1, \diamond}^{1}(\Omega), \quad \text { and } \quad \tilde{\theta}:=-\omega \in \widetilde{\mathrm{H}}_{1, \diamond}^{1}(\Omega),
$$

where $\hat{c}$ is a positive constant which will be specified later. Therefore, from the definition of bilinear form $\mathcal{A}(\cdot, \cdot)$ we obtain

$$
\begin{aligned}
\mathcal{A}((\psi, \omega),(\tilde{\varphi}, \tilde{\theta}))= & \left(\sigma \operatorname{curl}_{\mathbf{a}} \psi, \operatorname{curl}_{\mathbf{a}} \tilde{\varphi}\right)_{r, \Omega}+\left(\sqrt{\nu} \operatorname{curl}_{\mathbf{a}} \omega, \operatorname{curl}_{\mathbf{a}} \tilde{\varphi}\right)_{r, \Omega} \\
& +\left(\sqrt{\nu} \operatorname{curl}_{\mathbf{a}} \tilde{\theta}, \operatorname{curl}_{\mathbf{a}} \psi\right)_{r, \Omega}-(\omega, \tilde{\theta})_{r, \Omega} \\
\geq & \sigma_{\min }\left\|\operatorname{curl}_{\mathbf{a}} \psi\right\|_{\mathrm{L}_{1}^{2}(\Omega)^{2}}^{2}+\hat{c}\left(\sqrt{\nu} \sigma \operatorname{curl}_{\mathbf{a}} \psi, \operatorname{curl}_{\mathbf{a}} \omega\right)_{r, \Omega} \\
& +\hat{c} \nu\left\|\operatorname{curl}_{\mathbf{a}} \omega\right\|_{\mathrm{L}_{1}^{2}(\Omega)^{2}}^{2}+\left(\sqrt{\nu} \operatorname{curl}_{\mathbf{a}} \psi, \operatorname{curl}_{\mathbf{a}} \omega\right)_{r, \Omega} \\
& -\left(\sqrt{\nu} \operatorname{curl}_{\mathbf{a}} \psi, \operatorname{curl}_{\mathbf{a}} \omega\right)_{r, \Omega}+\|\omega\|_{\mathrm{L}_{1}^{2}(\Omega)}^{2} \\
\geq & \sigma_{\min }\left\|\operatorname{curl}_{\mathbf{a}} \psi\right\|_{\mathrm{L}_{1}^{2}(\Omega)^{2}}^{2}-\frac{\hat{c}^{2} \sigma_{\max }^{2}}{2 \sigma_{\min }} \nu \operatorname{curl}_{\mathbf{a}} \omega \|_{\mathrm{L}_{1}^{2}(\Omega)^{2}}^{2} \\
& -\frac{\sigma_{\min }}{2}\left\|\operatorname{curl}_{\mathbf{a}} \psi\right\|_{\mathrm{L}_{1}^{2}(\Omega)^{2}}^{2}+\hat{c} \nu\left\|\operatorname{curl}_{\mathbf{a}} \omega\right\|_{\mathrm{L}_{1}^{2}(\Omega)^{2}}^{2}+\|\omega\|_{\mathrm{L}_{1}^{2}(\Omega)}^{2} \\
= & \frac{\sigma_{\min }}{2}\left\|\operatorname{curl}_{\mathbf{a}} \psi\right\|_{\mathrm{L}_{1}^{2}(\Omega)^{2}}^{2}+\hat{c}\left(1-\frac{\hat{c} \sigma_{\max }^{2}}{2 \sigma_{\min }}\right) \nu\left\|\operatorname{curl}_{\mathbf{a}} \omega\right\|_{\mathrm{L}_{1}^{2}(\Omega)^{2}}^{2} \\
& +\|\omega\|_{\mathrm{L}_{1}^{2}(\Omega)}^{2},
\end{aligned}
$$


and choosing $\hat{c}=\frac{\sigma_{\min }}{\sigma_{\max }^{2}}$, we can assert that

$$
\mathcal{A}((\psi, \omega),(\tilde{\varphi}, \tilde{\theta})) \geq C\|(\psi, \omega)\|_{\mathcal{X}}^{2},
$$

with $C$ independent of $\nu$, where we have used 2.5 and $(2.6)$ to derive the last inequality. On the other hand,

$$
\|\tilde{\theta}\|_{\widetilde{\mathrm{H}}_{1}^{1}(\Omega)}=\|\omega\|_{\widetilde{\mathrm{H}}_{1}^{1}(\Omega)} \quad \text { and } \quad\|\tilde{\varphi}\|_{\widetilde{\mathrm{H}}_{1}^{1}(\Omega)} \leq C\left(\|\psi\|_{\widetilde{\mathrm{H}}_{1}^{1}(\Omega)}+\|\omega\|_{\widetilde{\mathrm{H}}_{1}^{1}(\Omega)}\right),
$$

and consequently

$$
\sup _{\substack{(\varphi, \theta) \in \mathcal{X} \\(\varphi, \theta) \neq 0}} \frac{\mathcal{A}((\psi, \omega),(\varphi, \theta))}{\|(\varphi, \theta)\|_{\mathcal{X}}} \geq \frac{\mathcal{A}((\psi, \omega),(\tilde{\varphi}, \tilde{\theta}))}{\|(\tilde{\varphi}, \tilde{\theta})\|_{\mathcal{X}}} \geq C\|(\psi, \omega)\|_{\mathcal{X}} \quad \forall(\psi, \omega) \in \mathcal{X}
$$

which gives 3.3 .

Remark 2 Vorticity and stream-function are available after solving (2.7). On the other hand, as a consequence of the Lax-Milgram Theorem, the pressure can be computed as the unique solution of the following problem: Find $p \in \mathrm{H}_{1}^{1}(\Omega) \cap \mathrm{L}_{1,0}^{2}(\Omega)$ such that

$$
(\nabla p, \nabla q)_{r, \Omega}=G^{\psi}(q):=\left(\boldsymbol{f}-\sigma \operatorname{curl}_{\mathbf{a}} \psi, \nabla q\right)_{r, \Omega} \quad \forall q \in \mathrm{H}_{1}^{1}(\Omega) \cap \mathrm{L}_{1,0}^{2}(\Omega) .
$$

Moreover, the following continuous dependence holds: there exists $C>0$ independent of $\nu$ such that

$$
\|p\|_{\mathrm{H}_{1}^{1}(\Omega) \cap \mathrm{L}_{1,0}^{2}(\Omega)} \leq C\|\boldsymbol{f}\|_{\mathrm{L}_{1}^{2}(\Omega)^{2}} .
$$

Notice that, according to Remark 1, if tangential velocity is imposed on $\Gamma_{t}$, or if non-homogeneous Dirichlet data are set for the vorticity, then $G^{\psi}(q)$ should be replaced by $G^{\psi, \omega}(q)=\left(\boldsymbol{f}-\sigma \mathbf{c u r l}_{\mathbf{a}} \psi-\right.$ $\left.\sqrt{\nu} \operatorname{curl}_{\mathbf{a}} \omega, \nabla q\right)_{r, \Omega}$ in $(3.5$. Analogously for the discrete problem (4.4).

\section{Mixed finite element approximation}

In this section, we construct discrete schemes associated to (2.7) and (3.5), define explicit finite element subspaces yielding its unique solvability, derive a priori error estimates and provide the rate of convergence of the methods.

\subsection{Statement of the Galerkin scheme}

Let $\left\{\mathcal{T}_{h}\right\}_{h>0}$ be a regular family of triangulations of $\Omega$ by triangles $T$ with mesh size $h$. For $S \subset \bar{\Omega}$, we denote by $\mathbb{P}_{k}(S), k \in \mathbb{N}$, the set of polynomials of degree $\leq k$. For any $k \geq 1$, we adopt the subspaces

$$
\begin{aligned}
\mathrm{Z}_{h} & :=\left\{\varphi_{h} \in \widetilde{\mathrm{H}}_{1, \diamond}^{1}(\Omega):\left.\varphi_{h}\right|_{T} \in \mathbb{P}_{k}(T) \forall T \in \mathcal{T}_{h}\right\} \\
\mathrm{Q}_{h} & :=\left\{q_{h} \in \mathrm{H}_{1}^{1}(\Omega):\left.q_{h}\right|_{T} \in \mathbb{P}_{k}(T) \forall T \in \mathcal{T}_{h}\right\} \cap \mathrm{L}_{1,0}^{2}(\Omega) .
\end{aligned}
$$

Then, the finite element discretization for (2.7) reads: Find $\left(\psi_{h}, \omega_{h}\right) \in \mathrm{Z}_{h} \times \mathrm{Z}_{h}$ such that

$$
\begin{array}{ll}
a\left(\psi_{h}, \varphi_{h}\right)+b\left(\varphi_{h}, \omega_{h}\right)=F\left(\varphi_{h}\right) & \forall \varphi_{h} \in \mathrm{Z}_{h}, \\
b\left(\psi_{h}, \theta_{h}\right)-d\left(\omega_{h}, \theta_{h}\right)=0 & \forall \theta_{h} \in \mathrm{Z}_{h} .
\end{array}
$$

In turn, the discrete counterpart of $(3.5)$ is: Find $p_{h} \in \mathrm{Q}_{h}$ such that

$$
\left(\nabla p_{h}, \nabla q_{h}\right)_{r, \Omega}=G^{\psi_{h}}\left(q_{h}\right):=\left(\boldsymbol{f}-\sigma \mathbf{c u r l}_{\mathbf{a}} \psi_{h}, \nabla q_{h}\right)_{r, \Omega} \quad \forall q_{h} \in \mathrm{Q}_{h} .
$$


4.2 Solvability and stability analysis

We now establish discrete counterparts of Theorem 2 and Remark 2 , which will yield the solvability and stability of problems (4.3) and (4.4). First we state a discrete version of Theorem 1.

Theorem 3 Let $\left(\mathcal{X},\langle\cdot, \cdot\rangle_{\mathcal{X}}\right)$ be a Hilbert space and let $\left\{\mathcal{X}_{h}\right\}_{h>0}$ be a sequence of finite-dimensional subspaces of $\mathcal{X}$. Let $\mathcal{A}: \mathcal{X} \times \mathcal{X} \rightarrow \mathbb{R}$ be a bounded symmetric bilinear form, and $\mathcal{G}: \mathcal{X} \rightarrow \mathbb{R}$ a bounded functional. Assume that there exists $\bar{\beta}_{h}>0$ such that

$$
\sup _{\substack{y_{h} \in \mathcal{X}_{h} \\ y \neq 0}} \frac{\mathcal{A}\left(x_{h}, y_{h}\right)}{\left\|y_{h}\right\|_{\mathcal{X}}} \geq \bar{\beta}_{h}\left\|x_{h}\right\|_{\mathcal{X}} \quad \forall x_{h} \in \mathcal{X}_{h} .
$$

Then, there exists a unique $x_{h} \in \mathcal{X}_{h}$, such that

$$
\mathcal{A}\left(x_{h}, y_{h}\right)=\mathcal{G}\left(y_{h}\right) \quad \forall y_{h} \in \mathcal{X}_{h}
$$

Moreover, there exist $C_{1}, C_{2}>0$, independent of the solution, such that

$$
\left\|x_{h}\right\|_{\mathcal{X}} \leq C_{1}\left\|\left.\mathcal{G}\right|_{\mathcal{X}_{h}}\right\|_{\mathcal{X}_{h}^{\prime}}, \quad \text { and } \quad\left\|x-x_{h}\right\|_{\mathcal{X}} \leq C_{2} \inf _{y_{h} \in \mathcal{X}_{h}}\left\|x-y_{h}\right\|_{\mathcal{X}},
$$

where $x \in \mathcal{X}$ is the unique solution of continuous problem 3.2 .

Proof The proof follows from Theorem 1, and from the discrete inf-sup condition for $\mathcal{A}(\cdot, \cdot)$.

The unique solvability and convergence of the discrete problem 4.3 are stated next.

Theorem 4 Let $k \geq 1$ be an integer and let $\mathrm{Z}_{h}$ be given by 4.1). Then, there exists a unique $\left(\psi_{h}, \omega_{h}\right) \in \mathrm{Z}_{h} \times \mathrm{Z}_{h}$ solution of discrete problem 4.3 . Moreover, there exist constants $\hat{C}_{1}, \hat{C}_{2}>0$ independent of $h$ and $\nu$, such that

$$
\left\|\psi_{h}\right\|_{\widetilde{\mathrm{H}}_{1}^{1}(\Omega)}+\left\|\omega_{h}\right\|_{\widetilde{\mathrm{H}}_{1}^{1}(\Omega)} \leq \hat{C}_{1}\|\boldsymbol{f}\|_{\mathrm{L}_{1}^{2}(\Omega)^{2}},
$$

and

$$
\begin{aligned}
\left\|\psi-\psi_{h}\right\|_{\widetilde{\mathrm{H}}_{1}^{1}(\Omega)} & +\left\|\omega-\omega_{h}\right\|_{\widetilde{\mathrm{H}}_{1}^{1}(\Omega)} \\
& \leq \hat{C}_{2} \inf _{\left(\varphi_{h}, \theta_{h}\right) \in \mathrm{Z}_{h} \times \mathrm{Z}_{h}}\left(\left\|\psi-\varphi_{h}\right\|_{\widetilde{\mathrm{H}}_{1}^{1}(\Omega)}+\left\|\omega-\theta_{h}\right\|_{\widetilde{\mathrm{H}}_{1}^{1}(\Omega)}\right),
\end{aligned}
$$

where $(\psi, \omega) \in \widetilde{\mathrm{H}}_{1}^{1}(\Omega) \times \widetilde{\mathrm{H}}_{1}^{1}(\Omega)$ is the unique solution to variational problem (2.7).

Proof We define $\mathcal{X}_{h}:=\mathrm{Z}_{h} \times \mathrm{Z}_{h}$ and we consider $\mathcal{A}(\cdot, \cdot)$ and $\mathcal{G}(\cdot)$ as in the proof of Theorem 2 , The next step consists in proving that the bilinear form $\mathcal{A}(\cdot, \cdot)$ satisfies the discrete inf-sup condition 4.5. In fact, given $\left(\psi_{h}, \omega_{h}\right) \in \mathcal{X}_{h}$, we define

$$
\tilde{\theta}_{h}:=-\omega_{h} \in \mathrm{Z}_{h}, \quad \text { and } \quad \tilde{\varphi}_{h}:=\left(\psi_{h}+\frac{\sigma_{\min }}{\sigma_{\max }^{2}} \sqrt{\nu} \omega_{h}\right) \in \mathrm{Z}_{h}
$$

We now establish the stability and approximation property for the discrete pressure.

Theorem 5 Let $k \geq 1$ be an integer and let $\mathrm{Q}_{h}$ be given by 4.2 . Then, there exists a unique solution $p_{h} \in \mathrm{Q}_{h}$ to discrete problem (4.4) and there exists a constant $C>0$ such that:

$$
\left\|p_{h}\right\|_{\mathrm{H}_{1}^{1}(\Omega) \cap \mathrm{L}_{1,0}^{2}(\Omega)} \leq C\|\boldsymbol{f}\|_{\mathrm{L}_{1}^{2}(\Omega)^{2}} .
$$


Moreover, there exists a constant $\widehat{C}>0$ such that

$$
\begin{aligned}
\left\|p-p_{h}\right\|_{\mathrm{H}_{1}^{1}(\Omega) \cap \mathrm{L}_{1,0}^{2}(\Omega)} \leq & \widehat{C}\left(\inf _{q_{h} \in \mathrm{Q}_{h}}\left\|p-q_{h}\right\|_{\mathrm{H}_{1}^{1}(\Omega) \cap \mathrm{L}_{1,0}^{2}(\Omega)}\right. \\
& \left.+\inf _{\varphi_{h}, \theta_{h} \in \mathrm{Z}_{h}}\left(\left\|\psi-\varphi_{h}\right\|_{\widetilde{\mathrm{H}}_{1}^{1}(\Omega)}+\left\|\omega-\theta_{h}\right\|_{\widetilde{\mathrm{H}}_{1}^{1}(\Omega)}\right)\right),
\end{aligned}
$$

where $C$ and $\widehat{C}$ are independent of $\nu$ and $h$, and $p \in \mathrm{H}_{1}^{1}(\Omega) \cap \mathrm{L}_{1,0}^{2}(\Omega)$ is the unique solution of problem (3.5).

Proof On the one hand, the well posedness of problem 4.4 follows from the Lax-Milgram Theorem. On the other hand, from the well-known first Strang Lemma, we have that

$$
\begin{aligned}
\left\|p-p_{h}\right\|_{\mathrm{H}_{1}^{1}(\Omega) \cap \mathrm{L}_{1,0}^{2}(\Omega)} \leq & C\left\{\inf _{q_{h} \in \mathrm{Q}_{h}}\left\|p-q_{h}\right\|_{\mathrm{H}_{1}^{1}(\Omega) \cap \mathrm{L}_{1,0}^{2}(\Omega)}\right. \\
& \left.+\sup _{q_{h} \in \mathrm{Q}_{h}} \frac{G^{\psi_{h}}\left(q_{h}\right)-G^{\psi}\left(q_{h}\right)}{\left\|q_{h}\right\|_{\mathrm{H}_{1}^{1}(\Omega) \cap \mathrm{L}_{1,0}^{2}(\Omega)}}\right\} .
\end{aligned}
$$

To estimate the second term on the right-hand side above, we use the definition of $G^{\psi}$ (cf. (3.5)) and $G^{\psi_{h}}$ (cf. (4.4) ) to obtain

$$
\sup _{q_{h} \in \mathrm{Q}_{h}} \frac{G^{\psi_{h}}\left(q_{h}\right)-G^{\psi}\left(q_{h}\right)}{\left\|q_{h}\right\|_{\mathrm{H}_{1}^{1}(\Omega) \cap \mathrm{L}_{1,0}^{2}(\Omega)}} \leq C\left\|\operatorname{curl}_{\mathbf{a}}\left(\psi-\psi_{h}\right)\right\|_{\mathrm{L}_{1}^{2}(\Omega)^{2}} \leq C\left\|\psi-\psi_{h}\right\|_{\widetilde{\mathrm{H}}_{1}^{1}(\Omega)},
$$

where in the last inequality we have used (2.6). Therefore, the proof follows from 4.8).

\subsection{Convergence analysis}

According to Theorems 4 and 5, it only remains to prove that $\psi, \omega$ and $p$ can be conveniently approximated by functions in $\mathrm{Z}_{h}$ and $\mathrm{Q}_{h}$, respectively. With this purpose, we introduce the Lagrange interpolation operator $\Pi_{h}: \widetilde{\mathrm{H}}_{1}^{1}(\Omega) \cap \mathrm{H}_{1}^{2}(\Omega) \rightarrow \mathrm{Z}_{h}$.

Lemma 4 There exists $C>0$, independent of $h$, such that for all $\varphi \in \mathrm{H}_{1}^{k+1}(\Omega)$ :

$$
\left\|\varphi-\Pi_{h} \varphi\right\|_{\widetilde{\mathrm{H}}_{1}^{1}(\Omega)} \leq C h^{k}\|\varphi\|_{\mathrm{H}_{1}^{k+1}(\Omega)} .
$$

We now turn to the statement of convergence properties of the discrete problem 4.3 .

Theorem 6 Let $k \geq 1$ be an integer and let $Z_{h}$ and $Q_{h}$ be given by 4.1) and (4.2), respectively. Let $(\psi, \omega) \in \widetilde{\mathrm{H}}_{1, \diamond}^{1}(\Omega) \times \widetilde{\mathrm{H}}_{1, \diamond}^{1}(\Omega)$ and $p \in \mathrm{H}_{1}^{1}(\Omega) \cap \mathrm{L}_{1,0}^{2}(\Omega)$ be the unique solutions to the continuous problems (2.7) and (3.5), and $\left(\psi_{h}, \omega_{h}\right) \in \mathrm{Z}_{h} \times \mathrm{Z}_{h}$ and $p_{h} \in \mathrm{Q}_{h}$ be the unique solutions to the discrete problems 4.3 and 4.4 , respectively. Assume that $\psi \in \mathrm{H}_{1}^{k+1}(\Omega), \omega \in \mathrm{H}_{1}^{k+1}(\Omega)$, and $p \in \mathrm{H}_{1}^{k+1}(\Omega)$. Then, the following error estimates hold

$$
\begin{aligned}
\left\|\psi-\psi_{h}\right\|_{\widetilde{\mathrm{H}}_{1}^{1}(\Omega)}+\left\|\omega-\omega_{h}\right\|_{\widetilde{\mathrm{H}}_{1}^{1}(\Omega)} \leq C_{1} h^{k}\left(\|\psi\|_{\mathrm{H}_{1}^{k+1}(\Omega)}+\|\omega\|_{\mathrm{H}_{1}^{k+1}(\Omega)}\right) \\
\left\|p-p_{h}\right\|_{\mathrm{H}_{1}^{1}(\Omega) \cap \mathrm{L}_{1,0}^{2}(\Omega)} \leq C_{2} h^{k}\left(\|p\|_{\mathrm{H}_{1}^{k+1}(\Omega)}+\|\psi\|_{\mathrm{H}_{1}^{k+1}(\Omega)}+\|\omega\|_{\mathrm{H}_{1}^{k+1}(\Omega)}\right) .
\end{aligned}
$$

where $C_{1}$ and $C_{2}$ are positive constants independent of $\nu$ and $h$.

Proof The proof follows from estimates 4.8, 4.9) and error estimates from Lemma 4 
A natural consequence of this result is that the vorticity and stream-function approximations also converge in the $\mathrm{L}_{1}^{2}(\Omega)$-norm with an order $O\left(h^{k}\right)$ :

$$
\left\|\omega-\omega_{h}\right\|_{L_{1}^{2}(\Omega)}=O\left(h^{k}\right), \quad \text { and } \quad\left\|\psi-\psi_{h}\right\|_{L_{1}^{2}(\Omega)}=O\left(h^{k}\right) .
$$

Such an estimate can be improved by one order of convergence, as show by the following theorem.

Theorem 7 Under the assumptions of Theorem 6 , there exists $C>0$ independent of $h$ and $\nu$ such that

$$
\begin{aligned}
& \left\|\omega-\omega_{h}\right\|_{\mathrm{L}_{1}^{2}(\Omega)} \leq C h^{k+1}\left(\|\psi\|_{\mathrm{H}_{1}^{k+1}(\Omega)}+\|\omega\|_{\mathrm{H}_{1}^{k+1}(\Omega)}\right) \\
& \left\|\psi-\psi_{h}\right\|_{\mathrm{L}_{1}^{2}(\Omega)} \leq C h^{k+1}\left(\|\psi\|_{\mathrm{H}_{1}^{k+1}(\Omega)}+\|\omega\|_{\mathrm{H}_{1}^{k+1}(\Omega)}\right)
\end{aligned}
$$

Proof The core of the proof is based on a duality argument. We first establish 4.10). Let us consider the following well-posed problem: Given $g \in \mathrm{L}_{1}^{2}(\Omega)$, find $(\rho, \xi) \in \widetilde{\mathrm{H}}_{1, \diamond}^{1}(\Omega) \times \widetilde{\mathrm{H}}_{1, \diamond}^{1}(\Omega)$ such that

$$
\begin{array}{ll}
a(\varphi, \rho)+b(\varphi, \xi)=0 & \forall \varphi \in \widetilde{\mathrm{H}}_{1, \diamond}^{1}(\Omega), \\
b(\rho, \theta)-d(\theta, \xi)=G(\theta) & \forall \theta \in \widetilde{\mathrm{H}}_{1, \diamond}^{1}(\Omega),
\end{array}
$$

where $G(\theta):=(g, \theta)_{r, \Omega}$. we will require the following regularity: $\rho \in \mathrm{H}_{1}^{2}(\Omega), \xi \in \mathrm{H}_{1}^{2}(\Omega)$. Moreover, we also assume that there exists a constant $C>0$, independent of $\nu$ and $g$ such that

$$
\|\rho\|_{\mathrm{H}_{1}^{2}(\Omega)}+\|\xi\|_{\mathrm{H}_{1}^{2}(\Omega)} \leq C\|g\|_{\mathrm{L}_{1}^{2}(\Omega)}
$$

Next, choosing $(\varphi, \theta)=\left(\psi-\psi_{h}, \omega-\omega_{h}\right)$ in 4.12 , we obtain

$$
\begin{aligned}
G\left(\omega-\omega_{h}\right) & =b\left(\rho, \omega-\omega_{h}\right)-d\left(\omega-\omega_{h}, \xi\right), \\
a\left(\psi-\psi_{h}, \rho\right)+b\left(\psi-\psi_{h}, \xi\right) & =0 .
\end{aligned}
$$

Moreover, from (2.7) and 4.3 we have that:

$$
\begin{aligned}
& b\left(\psi-\psi_{h}, \xi_{h}\right)-d\left(\omega-\omega_{h}, \xi_{h}\right)=0, \\
& a\left(\psi-\psi_{h}, \rho_{h}\right)+b\left(\rho_{h}, \omega-\omega_{h}\right)=0 .
\end{aligned}
$$

Thus, subtracting the above equations and 4.15 from 4.14, we obtain

$$
\begin{aligned}
G\left(\omega-\omega_{h}\right)= & b\left(\rho, \omega-\omega_{h}\right)-d\left(\omega-\omega_{h}, \xi\right)-b\left(\psi-\psi_{h}, \xi_{h}\right)+d\left(\omega-\omega_{h}, \xi_{h}\right) \\
& -a\left(\psi-\psi_{h}, \rho_{h}\right)-b\left(\rho_{h}, \omega-\omega_{h}\right)+a\left(\psi-\psi_{h}, \rho\right)+b\left(\psi-\psi_{h}, \xi\right) \\
= & b\left(\rho-\rho_{h}, \omega-\omega_{h}\right)-d\left(\omega-\omega_{h}, \xi-\xi_{h}\right)+b\left(\psi-\psi_{h}, \xi-\xi_{h}\right)+a\left(\psi-\psi_{h}, \rho-\rho_{h}\right),
\end{aligned}
$$

for all $\left(\rho_{h}, \xi_{h}\right) \in \mathrm{Z}_{h} \times \mathrm{Z}_{h}$. Hence,

$$
\begin{aligned}
\left|G\left(\omega-\omega_{h}\right)\right| \leq & C\left(\left\|\rho-\rho_{h}\right\|_{\widetilde{\mathrm{H}}_{1}^{1}(\Omega)}\left\|\omega-\omega_{h}\right\|_{\widetilde{\mathrm{H}}_{1}^{1}(\Omega)}+\left\|\omega-\omega_{h}\right\|_{L_{1}^{2}(\Omega)}\left\|\xi-\xi_{h}\right\|_{\mathrm{L}_{1}^{2}(\Omega)}\right. \\
& \left.+\left\|\psi-\psi_{h}\right\|_{\widetilde{\mathrm{H}}_{1}^{1}(\Omega)}\left\|\xi-\xi_{h}\right\|_{\widetilde{\mathrm{H}}_{1}^{1}(\Omega)}+\left\|\psi-\psi_{h}\right\|_{\widetilde{\mathrm{H}}_{1}^{1}(\Omega)}\left\|\rho-\rho_{h}\right\|_{\widetilde{\mathrm{H}}_{1}^{1}(\Omega)}\right) \\
\leq & C h^{k}\left(\|\psi\|_{\mathrm{H}_{1}^{k+1}(\Omega)}+\|\omega\|_{\mathrm{H}_{1}^{k+1}(\Omega)}\right)\left(\left\|\rho-\rho_{h}\right\|_{\widetilde{\mathrm{H}}_{1}^{1}(\Omega)}+\left\|\xi-\xi_{h}\right\|_{\widetilde{\mathrm{H}}_{1}^{1}(\Omega)}\right),
\end{aligned}
$$

for all $\left(\rho_{h}, \xi_{h}\right) \in \mathrm{Z}_{h} \times \mathrm{Z}_{h}$, where in the last inequality we have utilized Theorem 6 . Taking in particular $\left(\rho_{h}, \xi_{h}\right)$ as the Lagrange interpolants of $(\rho, \xi)$ (see Lemma 4), and then using the additional regularity result 4.13 in the above estimate, we obtain:

$$
\left|G\left(\omega-\omega_{h}\right)\right| \leq C h^{k+1}\left(\|\psi\|_{\mathrm{H}_{1}^{k+1}(\Omega)}+\|\omega\|_{\mathrm{H}_{1}^{k+1}(\Omega)}\right)\|g\|_{\mathrm{L}_{1}^{2}(\Omega)} .
$$



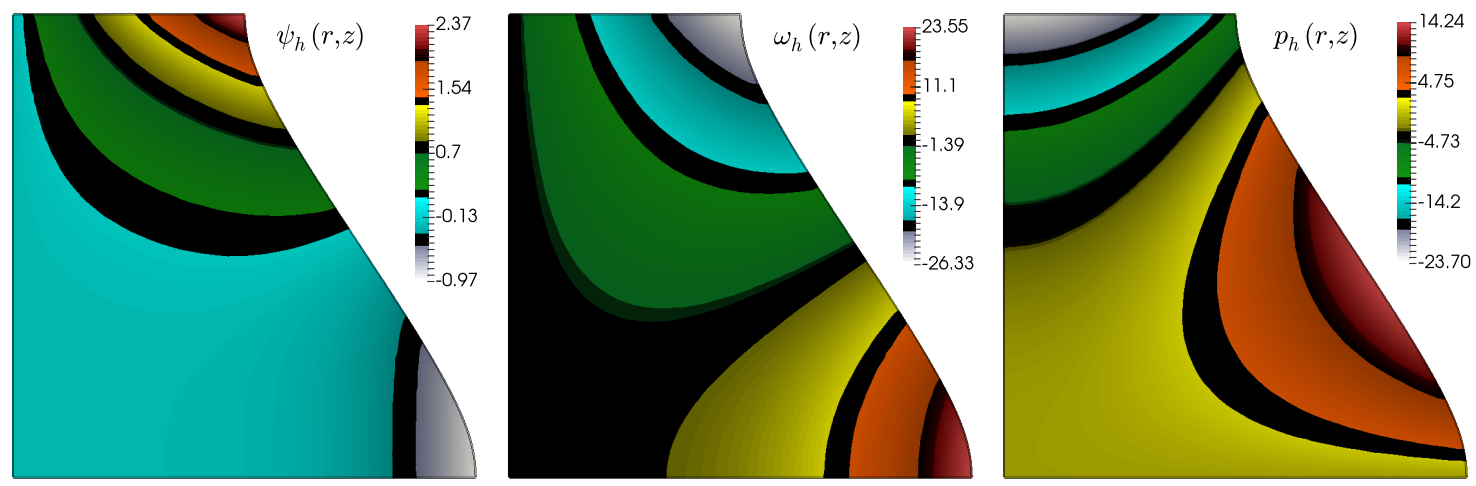

Fig. 1 Example 1: approximated stream-function, vorticity, and pressure distribution for the accuracy assessment test on the axisymmetric domain $\Omega$.

Thus, from the estimate above and the definition by duality of $\|\cdot\|_{L_{1}^{2}(\Omega)}$, we arrive at

$$
\left\|\omega-\omega_{h}\right\|_{\mathrm{L}_{1}^{2}(\Omega)}=\sup _{g \in \mathrm{L}_{1}^{2}(\Omega)} \frac{\left(g, \omega-\omega_{h}\right)_{r, \Omega}}{\|\left. g\right|_{\mathrm{L}_{1}^{2}(\Omega)}} \leq C h^{k+1}\left(\|\psi\|_{\mathrm{H}_{1}^{k+1}(\Omega)}+\|\omega\|_{\mathrm{H}_{1}^{k+1}(\Omega)}\right),
$$

where the constant $C$ is independent of $h$ and $\nu$.

Finally, (4.11) follows from the same arguments given before for 4.10, but instead of dual problem 4.12), we consider the following one:

$$
\begin{array}{ll}
a(\varphi, \rho)+b(\varphi, \xi)=G(\varphi) & \forall \varphi \in \widetilde{\mathrm{H}}_{1, \diamond}^{1}(\Omega), \\
b(\rho, \theta)-d(\theta, \xi)=0 & \forall \theta \in \widetilde{\mathrm{H}}_{1, \diamond}^{1}(\Omega),
\end{array}
$$

where in this case $G(\varphi):=(g, \varphi)_{r, \Omega}$.

Remark 3 We observe that since $\boldsymbol{u}=\operatorname{curl}_{\mathbf{a}} \psi$, the velocity can be readily recovered from the main unknowns of the underlying problem. More precisely, if $\left(\psi_{h}, \omega_{h}\right) \in \mathrm{Z}_{h} \times \mathrm{Z}_{h}$ is the unique solution of 4.3, then $\boldsymbol{u}_{h}:=\operatorname{curl}_{\mathbf{a}} \psi_{h}$ approximates the velocity with the same order of the proposed method. This result is summarized as follows.

Corollary 1 Assume that the hypotheses of Theorem 6 hold. Then, , there exists $C>0$ (independent of both $h$ and $\nu$ ) such that

$$
\left\|\boldsymbol{u}-\boldsymbol{u}_{h}\right\|_{\mathbf{H}\left(\operatorname{div}_{\mathrm{a}}, \Omega\right)} \leq C h^{k}\left(\|\psi\|_{\mathrm{H}_{1}^{k+1}(\Omega)}+\|\omega\|_{\mathrm{H}_{1}^{k+1}(\Omega)}\right) .
$$

Proof We have that

$$
\left\|\boldsymbol{u}-\boldsymbol{u}_{h}\right\|_{\mathbf{H}\left(\mathrm{div}_{\mathrm{a}}, \Omega\right)}=\left\|\boldsymbol{u}-\boldsymbol{u}_{h}\right\|_{\mathrm{L}_{1}^{2}(\Omega)^{2}}
$$

where in the last inequality we have used (2.6). Thus, the result follows from Theorem 6 .

\section{Numerical results}

In our first example we test the convergence of the proposed scheme when applied to the axisymmetric version of the classical colliding flow problem (see e.g. [19, Sect. 5.1] for the Cartesian case). The analytic solution is given as follows

$$
\psi(r, z)=5 r z^{4}-r^{5}, \quad \omega(r, z)=12 \sqrt{\nu}\left(2 r^{3}-5 r z^{2}\right), \quad p(r, z)=60 r^{2} z-24 z^{3},
$$


Table 1 Example 1: errors and convergence rates associated to the piecewise polynomial approximation of streamfunction, vorticity and pressure,

Table 2

and it is defined on the meridional domain $\Omega$ having four sides defined by the symmetry axis (left wall $r=0$ ), bottom and top lids ( $z=0$ and $z=1$, respectively), and the curve characterized by the parametrization $s \in[0,1], r=1-s / 2+0.15 \cos (\pi s) \sin (\pi s)$, and $z=s-0.15 \cos (\pi s) \sin (\pi s)$. We set the model parameters to $\sigma=10$ and $\nu=0.1$. The boundary conditions are non-homogeneous and set according to the interpolant of the exact stream-function and vorticity (and the pressure solve is modified according to Remark 21, whereas the forcing term $f$ has been manufactured using the momentum equation (??). Errors for vorticity and stream-function were measured in the $\widetilde{\mathrm{H}}_{1}^{1}(\Omega)$ and $\mathrm{L}_{1}^{2}(\Omega)$-norms (denoted hereafter with subscripts 1 and 0 , respectively), while those for the pressure correspond to the $\mathrm{H}_{1}^{1}(\Omega) \cap \mathrm{L}_{1,0}^{2}(\Omega)$-norm (denoted with subscript 1 ). The convergence history (obtained on a family of successively refined unstructured partitions of $\Omega$ ) is collected in Table 1 . confirming the expected behavior predicted by Theorems 6 and 7 . The approximate solutions obtained using the lowest-order method $(k=1)$ on a coarse mesh are displayed in Figure 1. We recall that, by construction, the divergence of the computed velocity is exactly zero.

Our next example addresses the well-known lid driven cavity flow. The domain under consideration is the unit square $\Omega=(0,1)^{2}$, discretized with an unstructured mesh of $80 \mathrm{~K}$ triangular elements. Following Remark 1 a tangential velocity $u^{t}=-1$ is imposed on the top lid of the domain $\left(\Gamma_{t} \subset \Gamma\right)$, we set homogeneous Dirichlet data for the stream-function. No boundary conditions are explicitly set for the vorticity. The forcing term is $\boldsymbol{f}=\mathbf{0}$, the viscosity is constant $\nu=1 e-2$, and the inverse permeability is, in a first round, constant $\sigma=0.1$. We also test the case where $\sigma$ is discontinuous across the line $r=0.4$, going from $\sigma_{0}=0.01$ to $\sigma_{1}=100$. Stream-function, vorticity and pressure profiles for both cases are displayed in Figure 2, where the bottom row shows a clear change of regime between the regions of high and low permeability.

Finally, we perform a simulation of axisymmetric laminar flow past a sphere. The meridional domain configuration is given in panel of Figure 3 . The boundary of the meridional $\Omega$ is decomposed into an inlet boundary (located at $z=0$ ), an outlet (at $z=10$ ), a "far-field" border (on $r=2$ ), the surface of the obstacle (centered at $r=0, z=5$ and with radius 1), and the symmetry axis is located at $r=0$. The domain is discretized into $80 \mathrm{~K}$ triangular elements and the model parameters are $\nu=5 e-3, \sigma=0.1$. The boundary conditions are set as follows: on $\Gamma_{\text {in }}$ we set $\psi=r$, on $\Gamma_{\text {far }}$ we set $\psi=\frac{1}{2} r^{2}$ and $\omega=0$, and on $\Gamma_{\text {obs }}$ we put $\psi=0$. The numerical results are depicted on the reflected domain in Figure 3, where we observe flow patterns qualitatively agreeing with the expected results (see e.g. [12]).

\section{Concluding remarks}

In the present paper, we have analyzed a mixed finite element method to approximate a streamfunction-vorticity variational formulation for the Brinkman problem in axisymmetric domains, which has been shown to be well-posed using standard arguments for mixed problems. The formulation was discretized by means of continuous piecewise polynomials of degree $k \geq 1$ for all the unknowns. We proved an $O\left(h^{k}\right)$ convergence with respect to the mesh size in the natural $H^{1}$-norm, as well as an $O\left(h^{k+1}\right)$ order in $L^{2}$-norm by a duality argument, and all estimates are uniform with respect to the fluid viscosity $\nu$. Finally, we reported numerical results that confirm the numerical analysis of the proposed method. A distinctive feature of this method is that it allows discrete velocities which are 

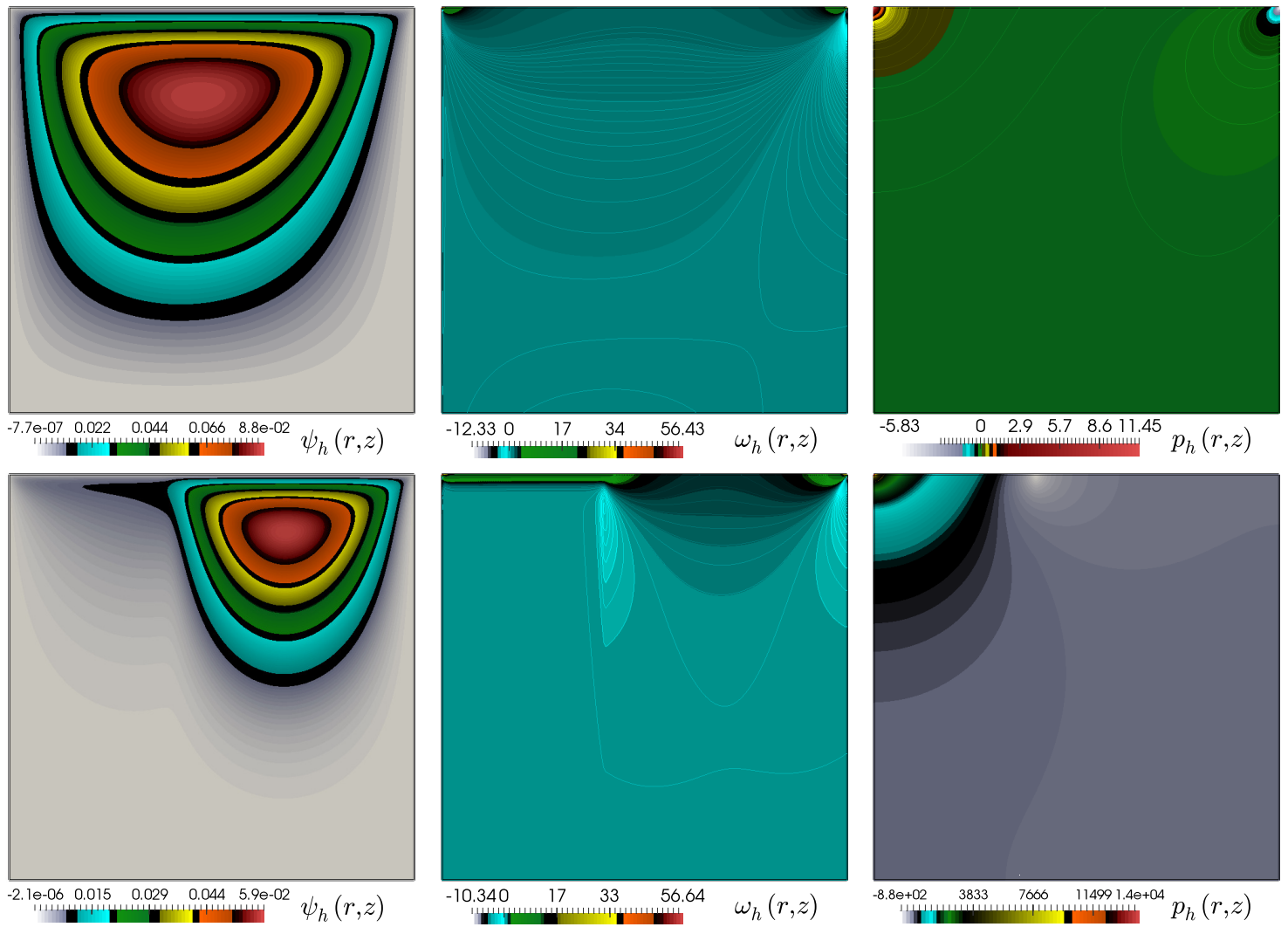

Fig. 2 Example 2: approximated stream-function, vorticity, and pressure distribution for the lid-driven cavity problem for constant (top row) and discontinuous permeability (bottom panels).

automatically divergence-free. Extensions of this work include the nonlinear Navier-Stokes equations and coupling with transport problems arising from multiphase flow descriptions.

Acknowledgements The first author was supported by DIUBB through project 151408 GI/VC. The second author was partially supported by CONICYT-Chile through FONDECYT project 1140791, by DIUBB through project 151408 GI/VC and by Anillo ANANUM, ACT1118, CONICYT (Chile). The fourth author acknowledges the support by the Elsevier Mathematical Sciences Sponsorship Fund MSSF-201602.

\section{References}

1.

3. N. Abdellatif, and C. Bernardi, A new formulation of the Stokes problem in a cylinder, and its spectral discretization. ESAIM: M2AN Math. Model. Numer. Anal., 38(5) (2004) 781-810.

4. N. Abdellatif, M. Touihri, and M. El Amin, Spectral element discretization for the stream-function and vorticity formulation of the axisymmetric Stokes problem. Calcolo, in press. DOI:10.1007/s10092-015-0152-1.

5. N. Abdellatif, N. Chorfi, and S. Trabelsi, Spectral discretization of the axisymmetric vorticity, velocity and pressure formulation of the Stokes problem. J. Sci. Comput., 47 (2011) 419-440.

6. V. Anaya, D. Mora, C. Reales, and R. Ruiz-Baier, Stabilized mixed approximation of axisymmetric Brinkman flows. ESAIM: Math. Model. Numer. Anal., 49(3) (2015) 855-874.

7. V. Anaya, D. Mora, and R. Ruiz-Baier, On a pure vorticity formulation for the Brinkman equations. $\mathrm{CI}^{2} \mathrm{MA}$ preprint 2015-21, available from www.ci2ma.udec.cl/publicaciones/prepublicaciones.

8. S.M. Aouadi, C. Bernardi, and J. Satouri, Mortar spectral element discretization of the Stokes problem in axisymmetric domains. Numer. Methods Part. Diff. Eqns., 30 (2014) 44-73.

9.

10. M. Azaiez, A spectral element projection scheme for incompressible flow with application to the unsteady axisymmetric Stokes problem. J. Sci. Comput., 17 (2002) 573-584. 


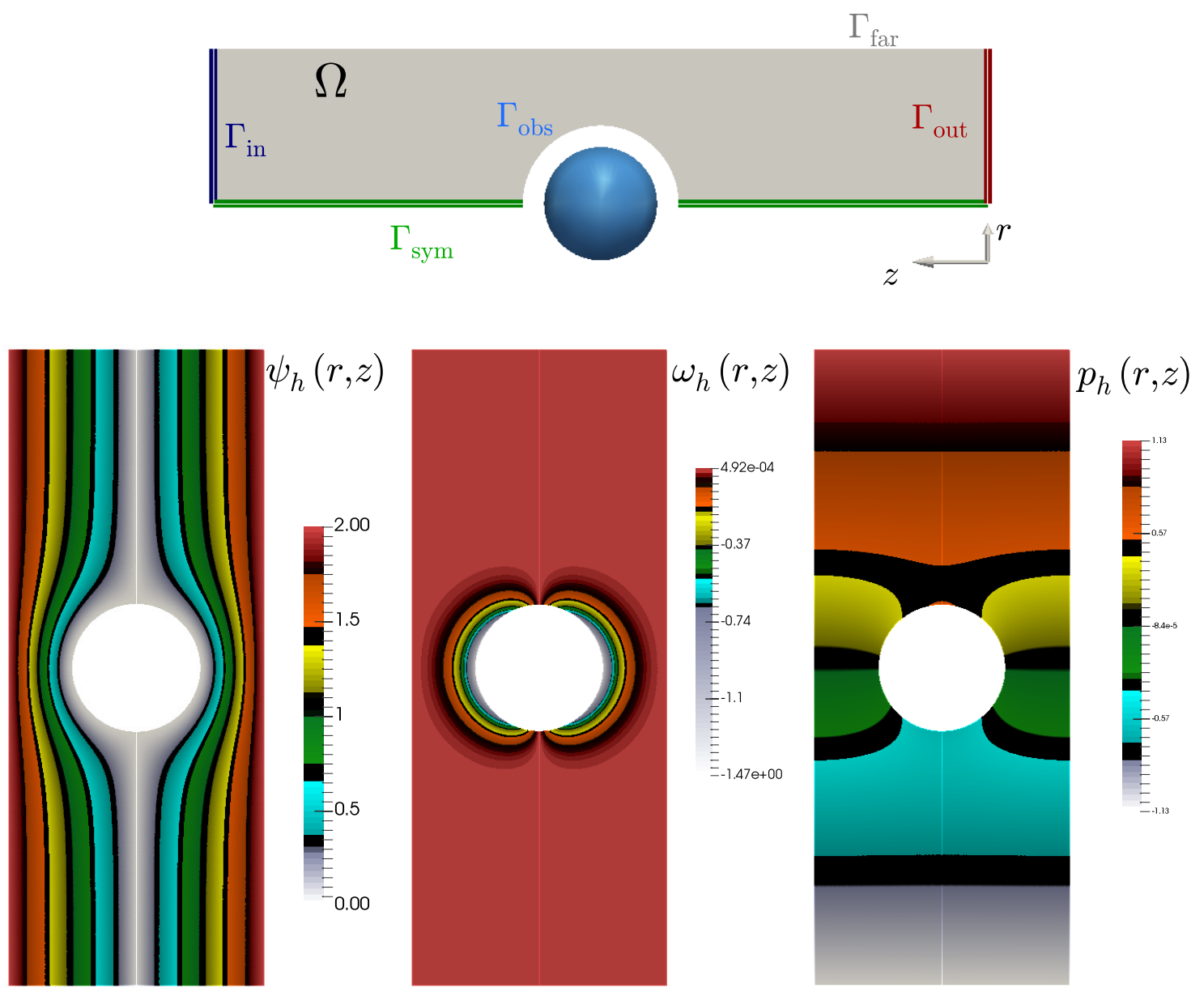

Fig. 3 Example 3: and approximate solutions for stream-function, vorticity, and pressure.

11. E. Barragy, and G.F. Carey, Stream function-vorticity driven cavity solutions using $p$ finite elements. Computers \& Fluids 26(5) (1997) 453-468.

12. G.K. Batchelor, An introduction to fluid dynamics. Cambridge University Press (1967).

13. C. Bègue, C. Conca, F. Murat, and O. Pironneau, Les équations de Stokes et de Navier-Stokes avec conditions aux limites sur la pression. In Nonlinear Partial Differential Equations and their applications, College de France Seminar, Ix, (1988) 179-264.

14. Z. Belhachmi, C. Bernardi, and S. Deparis, Weighted Clément operator and application to the finite element discretization of the axisymmetric Stokes problem. Numer. Math., 105(2) (2006) 217-247.

15. C. Bernardi, M. Dauge, and Y. Maday, Spectral methods for axisymmetric domains. Gauthier-Villars, Éditions Scientifiques et Médicales Elsevier, Paris, 1999.

16. J.H. Carneiro de Araujo, and V. Ruas, A stable finite element method for the axisymmetric three-field Stokes 17 . system. Comput. Methods Appl. Mech. Engrg. 164 (1998) 267-286.

18.

19. H. Elman, D. Silvester, and A. Wathen, Finite elements and fast iterative solvers, Oxford University Press, Oxford, 2005

20. V.J. Ervin, Approximation of coupled Stokes-Darcy flow in an axisymmetric domain. Comput. Methods Appl. Mech. Engrg. 258 (2013) 96-108.

21. G.N. Gatica, A Simple Introduction to the mixed finite element method. Theory and applications. Springer Briefs in Mathematics, Springer, Cham Heidelberg New York Dordrecht London, 2014.

22. G.N. Gatica, L.F. Gatica, and A. Márquez, Analysis of a pseudostress-based mixed finite element method for the Brinkman model of porous media flow. Numer. Math., 126(4) (2014) 635-677.

23. F. Ghadi, V. Ruas, and M. Wakrim, A mixed finite element method to solve the Stokes problem in the stream function and vorticity formulation. Hiroshima Math. J., 28 (1998) 381-398.

24. V. Girault, and P.A. Raviart, Finite element methods for Navier-Stokes equations. Theory and algorithms. Springer-Verlag, Berlin, 1986. 
25. J. Gopalakrishnan, and J. Pasciak, The convergence of V-cycle multigrid algorithms for axisymmetric Laplace and Maxwell equations. Math. Comp., 75 (2006) 1697-1719.

26. A. Kufner, Weighted Sobolev spaces. Wiley, New York, 1983.

27.

28

29. B. Mercier, and G. Raugel, Resolution d'un problème aux limites dans un ouvert axisymétrique par éléments finis en $r, z$ et séries de Fourier en $\theta$. RAIRO Anal. Numér., 16 (1982) 405-461.

30.

31.

32 .

33. V. Ruas, Mixed finite element methods with discontinuous pressures for the axisymmetric Stokes problem. ZAMM Z. Angew. Math. Mech., 83 (2003) 249-264.

34. T.E. Tezduyar, and J. Liou, On the downstream boundary conditions for the vorticity-stream function formulation of two-dimensional incompressible flows. Comput. Methods Appl. Mech. Engrg. 85 (1991) $207-217$.

35 . 